\title{
A different olfactory perception in anosmic patients: evidence from functional MRI
}

Mohsen Kohan Pour ${ }^{1}$, Sobhan Aarabi ${ }^{2}$, Seyed Amir Hossein Batouli ${ }^{3,1}$, Soodeh Moallemian ${ }^{1}$, Mohammad Ali Oghabian $4,1, *$

1 Neuroimaging and Analysis Group, Research Center for Molecular and Cellular Imaging, Tehran University of Medical Sciences, Tehran, Iran

2 Comprehensive Epilepsy Program, Epilepsy Monitoring Unit, Pars Hospital, Tehran, Iran

3 Department of Neuroscience and Addiction Studies, School of Advanced Technologies in Medicine, Tehran University of Medical Sciences, Tehran, Iran

4 Department of Medical Physics and Biomedical Engineering, School of Medicine, Tehran University of Medical Science, Tehran, Iran.

* Corresponding author: Prof. Mohammad Ali Oghabian; Department of Medical Physics and Biomedical Engineering, School of Medicine, Tehran University of Medical Science, Tehran, Iran; Email: oghabian@,sina.tums.ac.ir

\begin{abstract}
Olfactory system is a vital sensory system in mammals, giving them the ability to connect with their environment. Anosmia, or the complete loss of olfaction ability, which could be caused by injuries, is an interesting topic for inspectors with the aim of diagnosing patients. Sniffing test is currently utilized to examine if an individual is suffering from anosmia; however, functional Magnetic Resonance Imaging (fMRI) provides unique information about the structure and function of the different areas of the human brain, and therefore this noninvasive method could be used as a tool to locate the olfactory-related regions of the brain. In this study, by recruiting 31 healthy and anosmic individuals, we investigated the neural BOLD responses in the olfactory cortices following two odor stimuli, rose and eucalyptus, by using a 3T MR scanner. Comparing the two groups, we observed a network of brain areas being more active in the normal individuals when smelling the odors. In addition, a number of brain areas also showed an activation decline during the odor stimuli, which is hypothesized as a resource allocation deactivation. This study illustrated alterations in the brain activity between the normal individuals and anosmic patients when smelling odors, and could potentially help for a better anosmia diagnosis in the future.
\end{abstract}

Key Words: Olfaction; functional MRI; Anosmia 


\section{Introduction}

The importance of the sense of smell in everyday life and evolution is quite clear[1]. From its hedonistic aspect of life and eating satisfaction to its survival aspect, which involves detecting danger in the environment or toxins and edibility of food, all indicate the importance of having intact olfactory sensation[2, 3]. The olfactory pathway and network in primates can be traced back to the primary regions including olfactory bulb to the entorhinal and piriform cortices of the temporal lobes, and also to the thalamus, hypothalamus, and parts of the limbic system[4]. In a normal person, as an odor reaches the nose, two groups of olfactory regions, including the primary olfactory cortex- piriform cortex, entorhinal cortex and amygdalae - and some secondary regions, are stimulated [11]. Propagation of the olfactory receptor and olfactory bulb pass through the primary olfactory cortex, wherein the smell is perceived and recognized. Over time, more olfactory projections are issued into the lateral olfactory regions, which include orbitofrontal cortex, insula, hippocampus, thalamus and hypothalamus [16].

Olfactory dysfunction can be divided into two groups in terms of severity. Hyposmia, which is a partially loss of olfactory ability, and anosmia which is applied to complete olfactory dysfunction that leads to inefficiency in daily functioning [5]. The most important causes of olfactory dysfunctions are congenital, infections, age-related disorders and traumatic disorders [6], and the latter is the subject of this study. Traumatic loss of olfactory sensation is relatively common in head trauma which occurs in 5 to $14.5 \%$ of the cases[7]. Some frontal and temporal lobe injuries might cause post-traumatic hyposmia. Even in severe injuries, a complete dysfunction of this system is likely to occur [6,7]. While finding precise methodologies for the early diagnosis of these diseases and other sensory modalities are of great interest to the researchers, olfactory system of the brain is still understudied $[2,3,4,5]$.

Hyposmia and anosmia are the partial or complete loss of the ability to smell. While common conditions, irritating the sense of smell such as allergies lead to a temporary anosmia, and more serious conditions that affect the brain nervous system, e.g. brain tumors or head trauma and in early stages of diseases such as Alzheimer and Parkinson, can cause permanent loss of this sense $[1,2,6,7]$. There are also some rare individuals who are born without a functional olfactory sense [22]. Previous studies on the frequency of this kind of disease show that slightly more than $20 \%$ of the population are affected with sense of smell deficiency, and as the population grows older, this rate increases [21].

Diagnosis of traumatic anosmia and its severity is important in terms of diagnostic-therapeutic interventions and especially in legal claims and compensation. Traditional methods of assessing olfactory disabilities include selfreporting and simple sniffing odors kits[8]. These methods have notable limitations. Because these psychophysiological methods are based on self-report, they require both an appropriate level of awareness and cognition for reliable cooperation of patients. Also, they can be misdiagnosed through malingering for compensation[9]. Although conventional Magnetic Resonance Imaging (MRI) diagnoses gross olfactory system damages, it has limitations on subtle lesions and obviously in distinguishing underpinning networks. Other Imaging modalities using metabolic rate changes such as Positron Emission Tomography (PET) scans and single-photon emission computed tomography (SPECT) are also used to assess patients with anosmia; however, fMRI is preferable in some respects due to a better temporal and spatial resolution than both modalities[10]. 
Functional Magnetic Resonance Imaging (fMRI) was utilized in the evaluation of olfactory system pathophysiology in recent years. The results of studies in this field have shown similarities but also inconsistencies, which are due to their differences in the task design, populations, types of anosmia, and the stimulations, and therefore a careful interpretation is needed. Perception of odors is achieved from combined olfactory and trigeminal system activation[11]. Olfactory system is involved in the quality of odors while trigeminal reflects sensations such as burning, temperature and spicy[12]. Studies demonstrated that pleasant or unpleasant odors recruit shared but also different regions of the brain. For example Rolls et al. declared that beside activation of primary olfactory regions in both pleasant and unpleasant odors, medio-rostral OFC is mostly activated in pleasant odor, while BOLD signal was shown in the left lateral OFC in an unpleasant odor stimulation[13]. In addition, other studies represented that OFC and amygdala were involved in odor valence[14, 15]. In contrast with pleasant odor, studies emphasized on activation of anterior cingulate (ACC) and paracingulate cortex encountering with unpleasant stimulation[11, 16]. Furthermore, body of the literature established ACC as a key area involved in painful simulation and pain processing[17-19]. While these findings showed distinct areas of the limbic system responsible for pleasant and unpleasant odor, a meta-analysis illustrated that limbic lobe activations are overlapped in both types of odors[20].

Given the above, one of the applications of fMRI might be its ability as a reliable neuroimaging biomarker distinguishing patients with post-traumatic anosmia from healthy people. There are still wide variety of controversies and debates on this area. Previous studies have shown that the major difference in brain activity between healthy individuals and patients is more prominent in the primary and secondary olfactory system, including piriform cortex, amygdala, orbitofrontal cortex (OFC), insula, and anterior cingulate cortex[9, 21-23]. Intertwining of olfactory and trigeminal pathway in smelling causes loss pf cerebral activation in anosmic patients in brain areas related to trigeminal and bimodal stimulation. Two fMRI studies reported diminished activation in the motor areas and cerebellum in anosmia during trigeminal or bimodal stimulation[11,24]. Early investigation on regions out of well-known piriformOFC circuit, showed activations in the inferior frontal gyrus, caudate nucleus and also cerebellum during unpleasant olfactory stimulation[25]. Being similar with the population of the current study, a recent study by Moon et al. on patients with trauma-induced anosmia showed that the brain activity was reduced in both the primary and secondary olfactory regions (specifically the orbitofrontal cortex) compared with healthy individuals. This finding was more in the unpleasant odor[9]. All in all, the utility of fMRI in traumatic anosmic patients is not clear yet, and this study is an attempt to shed light on fMRI utilization and limitation for clinical diagnosis of anosmic patients.

This study was undertaken to find and compare regions of the brain activated while performing rose and eucalyptus odor stimuli in two groups of healthy and patient individuals using MR scanner. We anticipated that in rose odor, based on the frequency of this flavor in our culture, we will detect more activation in the healthy subjects. Furthermore, for this odor, memory-related areas of the brain such as amygdala might be more evoked, as our patients know what odor they are perceiving. For this aim, we recruited 31 participants and performed fMRI scan on them using a $3 \mathrm{~T}$ scanner, while presenting two odors to them. The results of this study could be a help for using fMRI as a diagnosis tool for anosmia, and it may also help for prediction purposes.

\section{Methods}




\subsection{Participants}

Sixteen anosmia patients with the mean age of 36.3 years old (range 22 to 50) were included in the study. An ENT (Ear, Nose, and Throat) specialist introduced us anosmia patients, from the “Amir Aalam” Hospital, Tehran, who were voluntarily referred for their olfaction disorder. In addition to the general examinations, a 'Sniff-in Sticks' test kit was also used, to estimate the threshold, discrimination, and identification (TDI) score, which is a reliable tool for measuring the function of the human olfactory system[26]. All patients had lost their sense of smell for at least two years, due to an injury, and none had undergone any pieces of training to recover their olfactory system. The second group included 15 healthy subjects (11M), with the mean age of 30.1 years old (range 20-39); their health criteria were assessed by a general practitioner.

This study was conducted based on the Ethics Statement of Tehran University of Medical Sciences which is in accordance with the principles of the revised Declaration of Helsinki. Informed consent was obtained after outlining the experimental paradigm in the first interview.

\subsection{Olfactory test}

Psychophysical testing of olfactory function was performed with the "Sniffing' Sticks" test (SST). In this test, odorants are presented in felt-tip pens. To perform the examination, the pen's tip is placed, approximately in $2 \mathrm{~cm}$ distance, in front of both nostrils for almost three seconds. The test includes three different scenarios to evaluate the acuity of the sense of smell. First, the odor threshold was investigated through a stepwise rarefaction of 16 N-butanol felt-tip pens. Then, the participants were asked to choose a target odor out of three odors presented to them by three different sticks, repeated for all 16 felt-tip pens. And finally, they were asked to identify 16 odorants in multi-choice lists of four felttip pens for each odor. Results from all three tests, ranging between 1 to 16, were summarized as the so-called TDI score. According to previous findings, we classified those participants with a TDI score of lower than 16 as anosmia, and the participants with TDI scores of above 30 were regarded healthy[27,28,46,48].

\subsection{Imaging}

The data were acquired using a 3T MRI scanner (MAGNETOM Trio; Siemens Healthcare GmbH, Federal Republic of Germany) using a standard receiving 32-channel head coil. Foam cushions were used to diminish the small head movements in the coil during scanning. Following a three dimensional magnetization, we were prepared with a rapid acquisition gradient echo sequence (repetition time $=1800 \mathrm{~ms}$, echo time $[\mathrm{TE}]=3.4 \mathrm{~ms}$, flip angle $=7$ degrees, field of view $[\mathrm{FOV}]=192 \mathrm{~mm} 2,176$ slices, slices thickness $=1 \mathrm{~mm}$, matrix size $=256 \times 256$, voxel size $=1 \times 1 \times 1 \mathrm{~mm} 3)$ to obtain high-resolution T1-weighted images of the brain. For fMRI analysis, an echo planar imaging (EPI), T2*weighted sequence with 755 whole-head volumes $(\mathrm{TR}=3000 \mathrm{~ms}$, TE $=30 \mathrm{~ms}, 40$ slices, no gap, flip angle $=90$ degress, $\mathrm{FOV}=192 \mathrm{~mm}^{2}$, slice thicknes $=3 \mathrm{~mm}$, and matrix size $\left.=64 \times 64\right)$ was acquired.

\section{4. fMRI paradigm}

Olfactory stimulation was administered using a computer-controlled stimulator (Mag-concept Olfactometer, 2010, USA). With a continuous airflow rate of $2 \mathrm{~L} / \mathrm{min}$, the olfactometer allows delivery of odor stimuli without altering relative conditions such as pressure and temperature, in the nasal cavity. It consists of three main parts: a positive air 
pressure, a nasal mask, and a delivery system consisting of six capsules containing the odorants. The olfactometer is designed so that it can be put in the scanner room as all of its components are made of diamagnetic materials. The participants were eyes-closed during the scan. This system was controlled by a computer program to permit choosing of, and switching between, different types of stimulation. Odor and air delivery timings, stimulus frequency, and choice of a specific odorant for stimulus presentation within any task were controlled by the software (Psychtoolbox MATLAB).

In this study, we used a block design protocol to perform our task. For each odor, the block consisted of two alternating sections: A 15-second odor presentation (e.g. eucalyptus; nature's alchemy Co. 100\% pure natural essential oil), followed by a rest period of 45 seconds (fragrance-free air). These patterns continued for 500 seconds (five complete rounds for each aroma). This protocol ran over for rose odorant as well. We used eucalyptus and rose odor in this study because of different nature of these odors in order to differentiate the pleasant and unpleasant odor stimulation and its related brain activation.

\subsection{Data Analysis}

\subsubsection{Preprocessing}

All of the analysis of fMRI data was performed by the fMRI Expert Analysis Tool (FEAT), part of FMRIB's Software Library (FSL, http://www.fmrib.ox.ac.uk/fsl) (version 5.0.9). First, we performed the Pre-processing steps including skull-stripping for removing all non-brain tissues using Brain Extraction Tool, FSL (BET); then motion correction was carried out via MCFLIRT, FSL (Motion Correction from FMRIB's Linear Image Registration Tool). Thereupon, slice-timing correction was accomplished with the help of Fourier-space time-series phase-shifting. Furthermore, normalization of the functional images to the standard Montreal Neurological Institute (MNI) brain atlas was fulfilled in two steps: Co-registering functional images of each participant to his/her high-resolution T1-weighted scan, with FLIRT (FMRIB's Linear Image Registration) and 7 degrees of freedom (DOF); and registering structural T1 images to the MNI space linearly, with 12 DOF. A Gaussian kernel of FWHM= $5.0 \mathrm{~mm}$ was used for spatial smoothing. Multiplicative mean intensity normalization of the volume at each time point was also performed. And finally, the data was filtered with high pass temporal filtering (Gaussian-weighted least-squares straight line fitting, with sigma = $60.0 \mathrm{~s})$.

\subsubsection{First-level analysis}

The General Linear Model (GLM) was performed using FEAT (version 6.0.0), FSL, for parametric statistical analysis. To make the statistical approaches valid and most efficient, a FILM (FMRIB Improved Linear Model) pre-whitening was utilized for statistical analysis of the fMRI time-series, devoting a "z-score" to the corresponding BOLD signal. In this method, a Boxcar function of tasks versus rest is created and convolved with a canonical hemodynamic response function and its temporal derivatives. As explained earlier, registration of the estimated functional map to the corresponding structural image and ultimately the MNI space were also carried out.

\subsubsection{Higher-level analysis}


Group-level analysis was performed under FLAME procedure (FMRIB's Local Analysis of Mixed Effects), to estimate within-group averages as well as between-group comparisons. A voxel-level probability threshold of zvalue $>2.6$ was applied on clusters to reveal significantly activated regions only. Also, a False Discovery Rate (p$\mathrm{FDR}<0.05$ ) was used to correct for multiple comparisons.

\section{Results}

\section{Brain activations in the healthy group}

Brain activations in healthy individuals during the eucalyptus and rose odor stimulations are provided in Table 1 and Figure 1. As shown in this table, on average, in our control group stimulated with eucalyptus odor, significant activations were observed in the well-known primary and secondary olfactory cortices, including piriform cortex (specifically in the left amygdala), left orbitofrontal cortex and left insula. In addition, regions related to emotion formation/regulation such as bilateral cingulate and paracingulate cortices were also activated. On the other hand, in the rose odor stimulation section, in addition to activating the same areas that were activated in the eucalyptus odor stimulation, the caudate area was also activated. In both odors, the activation of the thalamus was observed. When performing a statistical test between the brain activation patterns of healthy individuals relevant to the two odors, we could not identify any statistically significant difference between the brain activations relevant to the rose and eucalyptus odors.

\section{Brain activations in anosmic patients}

Our statistical analyses for averaging the brain activation maps of the anosmic patients, relevant to the two odors, showed that no brain regions survived this analysis, and therefore we could not identify any brain activations in this group.

\section{Comparison of normal and anosmic groups}

As provided in Table 1, we compared the normal and anosmic groups in their brain activations in response to the two odors. The normal >anosmic condition shows the brain areas with a higher activation in normals; however, as we had not observed any activation for the average of the anosmic group, the normal<anosmic group shows the deactivations of brain areas in the normal individuals during the odor stimuli.

A number of brain regions showed a significant activation in the normal $>$ anosmic condition, including bilateral (B) cerebellum, R amygdala, B inferior frontal gyrus, R orbitofrontal cortex, B insula, R frontal pole, L middle frontal gyrus, $\mathrm{R}$ frontal operculum, and $\mathrm{R}$ putamen. On the other hand, the following brain regions showed a lower activation in the normal group, which is interpreted as a deactivation in those brain areas: $\mathrm{L}$ precuneus, $\mathrm{L}$ angular gyrus, $\mathrm{L}$ superior frontal, B postcentral, B precentral, B cingulate, R superior frontal, L superior and L middle temporal gyri, as well as the $\mathrm{R}$ hippocampus.

\section{Discussions}

This study was designed to distinguish the brain activity in patients with post-traumatic olfactory dysfunction from the normal population, using the fMRI data during the odor presentation. In comparison with the healthy group, patients showed no activation in the primary nor secondary brain areas, either in stimulation with bimodal odor 
(eucalyptus) or unimodal (rose). There were a number of brain areas higher active in normal individuals during the odor stimuli, as well as a number of brain areas which showed deactivations during the stimuli, which will be discussed below.

We found the brain networks which showed activations in response to the two odors in the normal groups, but those networks did not show statistically significant differences. Our findings in the normal group on pleasant and unpleasant odors were similar to previous studies. In stimulation with both odors, the areas associated with the primary and secondary olfactory centers were activated. Compared to the smell of roses (pleasant), the smell of eucalyptus (bimodal), areas of cortical cingulate and paracingulate were activated, which was in line with the previous studies. This could be explained by the well-known role of the cingulate cortex in painful stimulation, and pain regulation should be noted[17-19]. We found the activation of left OFC in the unpleasant odor stimulation which was confirmed with the previous study[25]. Overall, OFC is related to the judgment of hedonic and aversive stimuli and its left side is mostly associated with aversive smell[2, 27]. It is notable to clarify that these differences are seen in average activation in both groups but there was no statistically meaningful difference in contrast between the two groups.

In the average of brain activations in the normal group, activation of the right supramarginal area was detected only in stimulation with eucalyptus in our results. This area is related to multisensory integration and it can be related to the bimodal nature of eucalyptus, and this activation was consistent with Frasnelii et al. study[28]. Most often early pieces of literature emphasized this claim that in contrast to all other sensations, there is no thalamic relay in the olfactory pathway to the neocortex[29].

In our study, activation of the thalamus in both odors was also recorded. Confirming our study, a recent study clarified the role of the thalamus in attention, discrimination, and learning of smells[30]. Thalamic pathway role in olfactory data processing was explored by Plaily et al. in a functional connectivity study. They demonstrated that piriformthalamus-OFC connectivity plays role in attention augmentation to odors[31]. However, the role of the thalamus in olfaction is still a debate.

Beside olfactory-related cortices, areas such as the superior and middle frontal were also activated in both odors. Areas that are known to be part of the ventral attention network, which is responsible for bottom-up sensory-driven exogenous attention and reorienting to unexpected external stimuli[32,33]. The activation of these regions is because of passive stimulation and the need to reorientation to unexpected stimuli (specific odor in this context). A considerable difference between eucalyptus and rose stimulation in control group was activation of the caudate nucleus in the rose group. Savic et al. showed that the caudate nucleus accompanied with ACC and prefrontal cortices participate in quality of odor than intensity[34]. On the other hand, caudate activation occurs in unexpected reward circuits and pleasure[35].

In the current study, inactivation was observed in primary and secondary olfactory cortex including amygdala, insula and OFC, which was consistent with the same study in Korea[9]. Our findings also supported the hypothesis about activation of the trigeminal nerve-related areas of the brain by pleasant odor which is declared in previous studies[9, 12]. These areas include the superior temporal gyrus, supramarginal gyrus and prefrontal cortex. Recruitment of the 
middle temporal gyrus is observed in a previous study in olfaction process which indicated multifunction nature of olfaction[9, 36]. Moon et al. also confirmed our findings in which an unpleasant odor (beta-mercaptoethanol) was used in their study, and they showed that this odor did not recruit trigeminal nerve-related cortex, and it seemed the unpleasant odor was more suitable for differentiating anosmic patients, than pleasant odor, because of false-positive effects of pleasant odor relating to trigeminal nerve activation[9].

Beyond the differences in primary and secondary olfactory cortices, areas including inferior frontal gyrus and cerebellum were also inactivated in the patients during stimulation with both odors (pleasant and unpleasant). These findings were supported with previous studies. Activation of the inferior frontal gyrus during smelling seems related to applying a specific name to the odor[25]. In addition, the role of cerebellum in smelling process is under question. We found inactivation of cerebellum in both side and in both odors which could be related to higher-order cognitive functions including language and memory, which are used in correct odor labeling[26].

This study provided supporting evidence for the fMRI's ability to diagnose and differentiate post-trauma anosmic patients from normal individuals, which emphasizes on the clinical use of neuroimaging in this area. Despite endeavors to perform a robust study, the work suffers from a number of limitations. First, a larger sample size would be more ideal. Second, performing the functional connectivity estimation would enrich our findings, which is a suggestion for the future works. Third, deactivation of the brain areas when being stimulated by an odor needs much more detailed interpretations. Finally, providing evidence on the brain activations when imagining an odor would help those centers with no access to the olfactometer to also perform an fMRI study on the human olfactory system.

\section{References}

1. Devanand, D.P., et al., Olfactory identification deficits and increased mortality in the community. Ann Neurol, 2015. 78(3): p. 401-11.

2. Pence, T.S., et al., Risk factors for hazardous events in olfactory-impaired patients. JAMA Otolaryngol Head Neck Surg, 2014. 140(10): p. 951-5.

3. Yeomans, M.R., Olfactory influences on appetite and satiety in humans. Physiol Behav, 2006. 87(4): p. 8004.

4. Fulbright, R.K., et al., Functional MR imaging of regional brain responses to pleasant and unpleasant odors. AJNR Am J Neuroradiol, 1998. 19(9): p. 1721-6.

5. Hummel, T., et al., Position paper on olfactory dysfunction. Rhinology, 2016. 56(1): p. 1-30.

6. Han, P., et al., Magnetic Resonance Imaging of Human Olfactory Dysfunction. Brain Topogr, 2019. 32(6): p. 987-997.

7. Costanzo, R.M. and T. Miwa, Posttraumatic olfactory loss. Adv Otorhinolaryngol, 2006. 63: p. 99-107.

8. Murphy, C., et al., Olfactory event-related potentials and aging: normative data. Int J Psychophysiol, 2000. 36(2): p. 133-45.

9. Moon, W.J., et al., Functional MRI as an Objective Measure of Olfaction Deficit in Patients with Traumatic Anosmia. AJNR Am J Neuroradiol, 2018. 39(12): p. 2320-2325. 
10. Kameyama, M., K. Murakami, and M. Jinzaki, Comparison of [(15)O] H2O Positron Emission Tomography and Functional Magnetic Resonance Imaging in Activation Studies. World J Nucl Med, 2016. 15(1): p. 3-6.

11. Iannilli, E., et al., Differences in anosmic and normosmic group in bimodal odorant perception: a functionalMRI study. Rhinology, 2011. 49(4): p. 458-63.

12. Albrecht, J., et al., The neuronal correlates of intranasal trigeminal function-an ALE meta-analysis of human functional brain imaging data. Brain Res Rev, 2010. 62(2): p. 183-96.

13. Rolls, E.T., M.L. Kringelbach, and I.E. de Araujo, Different representations of pleasant and unpleasant odours in the human brain. Eur J Neurosci, 2003. 18(3): p. 695-703.

14. Anderson, A.K., et al., Dissociated neural representations of intensity and valence in human olfaction. Nat Neurosci, 2003. 6(2): p. 196-202.

15. Chikazoe, J., et al., Population coding of affect across stimuli, modalities and individuals. Nature Neuroscience, 2014. 17(8): p. 1114-1122.

16. Bensafi, M., et al., Neural coding of stimulus concentration in the human olfactory and intranasal trigeminal systems. Neuroscience, 2008. 154(2): p. 832-8.

17. Apkarian, A.V., et al., Human brain mechanisms of pain perception and regulation in health and disease. Eur J Pain, 2005. 9(4): p. 463-84.

18. Yesudas, E. and T. Lee, The Role of Cingulate Cortex in Vicarious Pain. BioMed Research International, 2015. 2015: p. 1-10.

19. Xiao, X. and Y.Q. Zhang, A new perspective on the anterior cingulate cortex and affective pain. Neurosci Biobehav Rev, 2018. 90: p. 200-211.

20. Lindquist, K.A., et al., The brain basis of emotion: a meta-analytic review. Behav Brain Sci, 2012. 35(3): p. 121-43.

21. Levy, L.M., et al., Rapid imaging of olfaction by functional MRI (fMRI): identification of presence and type of hyposmia. J Comput Assist Tomogr, 1999. 23(5): p. 767-75.

22. Pellegrino, R., et al., Olfactory function in patients with hyposmia compared to healthy subjects - An fMRI study. Rhinology, 2016. 54(4): p. 374-381.

23. Han, P., et al., Impaired brain response to odors in patients with varied severity of olfactory loss after traumatic brain injury. J Neurol, 2018. 265(10): p. 2322-2332.

24. Iannilli, E., et al., Intranasal trigeminal function in subjects with and without an intact sense of smell. Brain Res, 2007. 1139: p. 235-44.

25. Smejkal, V., R. Druga, and J. Tintera, Olfactory activity in the human brain identified by fMRI. Bratisl Lek Listy, 2003. 104(6): p. 184-8.

26. Wabnegger, A. and A. Schienle, Cerebellar Gray Matter and Olfactory Performance. Chemical Senses, 2019. 44(7): p. 507-510.

27. Zald, D.H. and J.V. Pardo, Emotion, olfaction, and the human amygdala: amygdala activation during aversive olfactory stimulation. Proc Natl Acad Sci U S A, 1997. 94(8): p. 4119-24.

28. Frasnelli, J., et al., Dual processing streams in chemosensory perception. Front Hum Neurosci, 2012. 6: p. 288.

29. Smythies, J., The functional neuroanatomy of awareness: with a focus on the role of various anatomical systems in the control of intermodal attention. Conscious Cogn, 1997. 6(4): p. 455-81. 
30. Courtiol, E. and D.A. Wilson, The olfactory thalamus: unanswered questions about the role of the mediodorsal thalamic nucleus in olfaction. Front Neural Circuits, 2015. 9: p. 49.

31. Plailly, J., et al., Attention to odor modulates thalamocortical connectivity in the human brain. J Neurosci, 2008. 28(20): p. 5257-67.

32. Chica, A.B., P. Bartolomeo, and J. Lupianez, Two cognitive and neural systems for endogenous and exogenous spatial attention. Behav Brain Res, 2013. 237: p. 107-23.

33. Corbetta, M., G. Patel, and G.L. Shulman, The reorienting system of the human brain: from environment to theory of mind. Neuron, 2008. 58(3): p. 306-24.

34. Savic, I., et al., Olfactory functions are mediated by parallel and hierarchical processing. Neuron, 2000. 26(3): p. 735-45.

35. Berns, G., A. Brooks, and M. Spivak, Scent of the Familiar: An fMRI Study of Canine Brain Responses to Familiar and Unfamiliar Human and Dog Odors. Behavioural processes, 2014. 110.

36. Tubaldi, F., et al., Smelling odors, understanding actions. Soc Neurosci, 2011. 6(1): p. 31-47. 


\begin{tabular}{|c|c|c|c|c|c|c|}
\hline \multirow[b]{2}{*}{ Brain Areas } & $\begin{array}{l}\text { Normal- } \\
\text { Average }\end{array}$ & $\begin{array}{l}\text { Eucalyptus odo } \\
\text { Normal> } \\
\text { Anosmic }\end{array}$ & $\begin{array}{l}\text { Anosmic }> \\
\text { Normal }\end{array}$ & $\begin{array}{l}\text { Normal- } \\
\text { Average }\end{array}$ & \begin{tabular}{l}
\multicolumn{1}{c}{ Rose odor } \\
Normal> \\
Anosmic
\end{tabular} & $\begin{array}{l}\text { Anosmic }> \\
\text { Normal }\end{array}$ \\
\hline & $\mathbf{Z} / \mathbf{x}, \mathbf{y}, \mathbf{Z}$ & $\mathbf{Z} / \mathbf{x}, \mathbf{y}, \mathbf{Z}$ & $\mathbf{Z} / \mathbf{x}, \mathbf{y}, \mathbf{Z}$ & $\mathbf{Z} / \mathbf{x}, \mathbf{y}, \mathbf{Z}$ & $\mathbf{Z} / \mathbf{x}, \mathbf{y}, \mathbf{Z}$ & $\mathbf{Z} / \mathbf{x}, \mathbf{y}, \mathbf{Z}$ \\
\hline L cerebellum & $\begin{array}{l}5.32 /-26,-62,- \\
22\end{array}$ & --- & --- & $5.68 /-8,-86,-24$ & $3.66 /-4,-70,-16$ & --- \\
\hline $\mathrm{R}$ cerebellum & $5.22 / 24,-62,-20$ & $4.1 / 22,-64,-20$ & --- & ---- & --- & --- \\
\hline L Amygdala & $4.11 /-22,-4,-14$ & -- & --- & $4.29 /-24,0,-14$ & --- & --- \\
\hline R Amygdala & --- & $3.55 / 18,0,-16$ & --- & --- & --- & --- \\
\hline L IFG & --- & --- & --- & --- & $3.82 /-42,30,16$ & --- \\
\hline R IFG & $6.05 / 58,14,0$ & $3.54 / 50,8,18$ & ---- & --- & --- & ---- \\
\hline L OFC & $4.05 /-20,6,-22$ & --- & --- & --- & --- & --- \\
\hline R OFC & --- & --- & --- & --- & $3.8 / 34,28,-6$ & --- \\
\hline L lingual G. & --- & --- & --- & $\begin{array}{l}5.19 /-10,-82,- \\
18\end{array}$ & --- & --- \\
\hline $\mathrm{L}$ insula & --- & $4.24 /-38,6,-14$ & --- & --- & $3.72 /-28,26,2$ & --- \\
\hline $\mathrm{R}$ insula & --- & $4.5 / 38,-2,2$ & --- & $4.45 / 34,30,6$ & $3.8 / 36,22,-2$ & --- \\
\hline L Thalamus & --- & --- & --- & $3.94 /-10,-4,10$ & --- & --- \\
\hline $\mathrm{R}$ thalamus & $4.9 / 12,-4,14$ & --- & --- & --- & --- & --- \\
\hline L Frontal pole & --- & --- & --- & $4.21 /-48,46,14$ & --- & --- \\
\hline R Frontal pole & --- & $3.98 / 44,42,10$ & --- & --- & --- & --- \\
\hline L Middle frontal gyrus & $3.53 /-42,40,16$ & --- & --- & $4.81 /-50,16,44$ & $4.06 /-40,28,24$ & --- \\
\hline R PSC & $4.32 / 66,-12,20$ & --- & --- & --- & --- & --- \\
\hline R Caudate & --- & --- & --- & $4.14 / 10,2,12$ & --- & $\begin{array}{ll}-- \\
\end{array}$ \\
\hline R Frontal Operculum & --- & --- & --- & --- & $3.54 / 42,22,-2$ & --- \\
\hline R Putamen & $\begin{array}{ll}--- \\
\end{array}$ & $3.63 / 28,0,-10$ & --- & --- & --- & --- \\
\hline L Precuneus & "--- & - & "3.19/2,-62,30 & ב--- & "--- & "--- \\
\hline L Angular Gyrus & --- & --- & $3.79 /-38,-58,30$ & --- & --- & --- \\
\hline L Sup Frontal Gyrus & $3.94 /-40,52,18$ & --- & $3.41 /-20,26,56$ & $4.35 /-4,18,52$ & --- & --- \\
\hline L Postcentral G. & --- & --- & $4.02 /-2,-26,66$ & --- & --- & $3.82 /-44,-22,58$ \\
\hline R postcentral G. & $4.32 / 66,-12,20$ & --- & $3.78 / 10,-46,66$ & --- & --- & --- \\
\hline L Precentral G. & --- & --- & $3.76 /-22,-10,64$ & --- & --- & $4.2 /-10,-22,44$ \\
\hline R precentral G. & $4.84 / 60,8,4$ & --- & -- & --- & --- & $4.18 / 12,-30,74$ \\
\hline Paracingulate Gyrus & $4.3 /-2,16,44$ & --- & --- & $4.33 /-2,14,52$ & --- & $5.3 / 4,50,16$ \\
\hline L Cingulate Gyrus & $3 /-4,22,28$ & --- & $3.58 /-2,38,-6$ & -- & --- & $3.98 /-2,-4,40$ \\
\hline R Cingulate Gyrus & $2.97 / 6,22,34$ & --- & $3.44 / 4,-50,20$ & --- & --- & --- \\
\hline R SFG & -- & --- & --- & --- & --- & $4.85 / 2,50,24$ \\
\hline L Sup. Temp. G. & --- & --- & $3.39 / 58,4,-14$ & --- & --- & $3.64 /-60,-24,4$ \\
\hline L Mid. Temp. & --- & --- & -- & --- & --- & $4.06 /-52,-2,-24$ \\
\hline R Hippocampus & --- & --- & --- & --- & --- & $3.5 / 28,-24,-14$ \\
\hline
\end{tabular}

Table 1. Activation of the brain areas when presenting the eucalyptus or rose odors. An average of the activations in the normal individuals, as well as the contrasts of normal $>$ anosmic and normal $<$ anosmic, are provided in the table for both odors. $Z=z-$ value of the activation; $\mathrm{x}, \mathrm{y}, \mathrm{z}=$ coordinates of the voxel with the highest $\mathrm{z}$-value in the MNI standard space; $\mathrm{R}=\mathrm{right} \mathrm{L}=$ left. $\mathrm{IFG}=$ inferior frontal gyrus; $\mathrm{OFC}=$ orbitofrontal cortex; $\mathrm{G}=$ gyrus; PSC: Primary somatosensory cortex; $\mathrm{Sup}=$ superior; $\mathrm{SFG}=$ superior frontal gyrus; Temp= temporal; $\mathrm{Mid}=$ middle. 